\title{
Erratum to: A case study on occurrence of an unusual structure in the sodium layer over Gadanki, India
}

\author{
Sumanta Sarkhel ${ }^{1,2,3,4^{*}}$, John D Mathews ${ }^{1}$, Shikha Raizada ${ }^{2}$, Ramanathan Sekar ${ }^{5}$, Dibyendu Chakrabarty ${ }^{5}$, \\ Amitava Guharay ${ }^{6}$, Geonhwa Jee ${ }^{3}$, Jeong-Han Kim³ ${ }^{3}$ Robert B Kerr², Geetha Ramkumar ${ }^{7}$, Sundararajan Sridharan ${ }^{8}$, \\ Qian Wu' ${ }^{9}$ Martin G Mlynczak ${ }^{10}$ and James M Russell ${ }^{11}$
}

\section{Erratum}

After publication, it was found in the text that the words 'Figure 6' and 'Figure 7' were printed in the reverse order in several places of the article by Sarkhel et al. (2015). The details are as follows:

Page 7, Right column, Last paragraph, Line 8: 'Figure 6a' should be read as 'Figure 7a'

Page 9, Left column, Last paragraph, Line 11: 'Figure 7' should be read as 'Figure 6'

Page 9, Right column, Last paragraph, Line 1: 'Figure 6' should be read as 'Figure 7'

Page 10, Left column, Line 6: 'Figure 6a' should be read as 'Figure 7a'

Page 10, Left column, Line 25: 'Figure 6a' should be read as 'Figure 7a'

Page 10, Left column, Line 33: 'Figure 6a' should be read as 'Figure 7a'

Page 10, Left column, Line 37: 'Figure 6b' should be read as 'Figure 6'

Page 10, Right column, Line 15: 'Figure 6a' should be read as 'Figure 7a'

Page 10, Right column, Line 19: 'Figure 6b' should be read as 'Figure $7 \mathrm{~b}$ '

Page 11, Left column, Section: Role of sporadic-E activity, Line 12: 'Figure 6a' should be read as 'Figure 7a'

\section{Author details}

${ }^{1}$ Radar Space Sciences Laboratory, 323 Electrical Engineering East, The Pennsylvania State University, University Park, PA, USA. ${ }^{2}$ Space and Atmospheric Sciences, Arecibo Observatory, Center for Geospace Studies, SRI International, Arecibo, Puerto Rico, USA. ${ }^{3}$ Division of Climate Change, Korea Polar Research Institute, Incheon 406-840, South Korea. ${ }^{4}$ Department of
Physics, Indian Institute of Technology Roorkee, Roorkee 247667 Uttarakhand, India. ${ }^{5}$ Space and Atmospheric Sciences Division, Physical Research Laboratory, Ahmedabad, India. ${ }^{6}$ National Institute for Space Research, São José dos Campos, São Paulo, Brazil. ${ }^{7}$ Space Physics Laboratory, Vikram Sarabhai Space Centre, Thiruvananthapuram, India. ${ }^{8}$ National Atmospheric Research Laboratory, Gadanki, India. ${ }^{9}$ High Altitude Observatory, National Center for Atmospheric Research, Boulder, CO, USA. ${ }^{10}$ Atmospheric

Sciences Division, NASA Langley Research Center, Hampton, VA, USA.

${ }^{11}$ Center for Atmospheric Sciences, Hampton University, 23 Tyler Street, Hampton, VA, USA

Published online: 07 September 2015

\section{Reference}

Sarkhel S, Mathews JD, Shikha R, Ramanathan S, Dibyendu C, Amitava G, Geonhwa J, Jeong-Han K, Kerr RB, Geetha R, Sundararajan S, Qian W, Mlynczak MG, Russell JM (2015) A case study on occurrence of an unusual structure in the sodium layer over Gadanki, India. Earth Planets Space 67:19. doi:10.1186/s40623-015-0183-5

\section{Submit your manuscript to a SpringerOpen ${ }^{\odot}$} journal and benefit from:

- Convenient online submission

- Rigorous peer review

- Immediate publication on acceptance

- Open access: articles freely available online

- High visibility within the field

- Retaining the copyright to your article

Submit your next manuscript at springeropen.com

"Correspondence: sarkhel.fph@iitr.ac.in
'Radar Space Sciences Laboratory, 323 Electrical Engineering East, The Pennsylvania State University, University Park, PA, USA

${ }^{2}$ Space and Atmospheric Sciences, Arecibo Observatory, Center for Geospace Studies, SRI International, Arecibo, Puerto Rico, USA 